\title{
BIOMATERIAL REAL CRACKS EVALUATION BY EDDY GURRENT TESTING METHOD
}

The paper deals with identification of real biomaterial's cracks using electromagnetic non-destructive sensing. Advanced types of the magnetic field sensors are used for this purpose. New modular eddy current probe is used for the evaluation. Harmonic excitation is used for the excitation of the eddy currents. The real biomaterial stress corrosion cracks (SCC) and fatigue cracks (FC) are inspected. Gained results are discussed and presented in the paper. The main aim of the paper is to compare the sensitivity of the various magnetic field sensors under the same conditions.

Keywords: Nondestructive evaluation; inductance coil sensor, fluxgate magnetometer; GMR sensor; AMR sensor, biomaterial stress-corrosion and fatique cracks.

\section{Introduction}

Increased $R \& D$ activities in the field of non-destructive evaluation (NDE) have been motivated by the need for precise evaluation of materials for the assessment of the expected life of system components. System health monitoring (SHM) and condition based maintenance (CBM) are of high interest nowadays in order to sustain safety, reliability and quality of various processes. They come out from the so-called damage tolerance approach where an inspected element is actively used up to a certain point beyond which the structural integrity of a device could be affected. CBM and SHM systems include three phases: detection, diagnosis and prediction. The first two phases are inherently associated with non-destructive evaluation (NDE) of materials. NDE of materials is based on numerous physical principles and phenomena [1] and [2]. NDE techniques are widely used in a range of industries for the inspection of various complex structures. The periodic inspection of components and devices ensures their safe, effective and long-term operation. New methods and devices are still being developed and designed to tackle gradually increasing demands for reliable detection and precise characterization of material discontinuities. Increased $R \& D$ activities in the field of non-destructive evaluation (NDE) have been motivated by the need for precise evaluation of cracks and flaws for the assessment of the expected life of mechanical components. Nowadays, evaluation of stress corrosion cracks (SCC) and fatigue cracks (FC) in real biological environment is one of the most important problems, Fig. 1. These cracks strongly affect the structural integrity of the material or biomaterial objects.
Biomaterials are materials used to manufacture prostheses, implants, and surgical instruments.
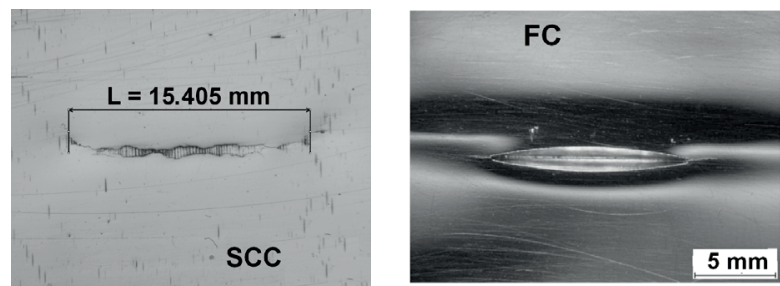

Fig. 1. Microscopy images of the inspected cracks

Designed not to provoke rejection by our bodies (skin, blood, bone, etc.), they can be natural (collagen, cellulose, etc.) or synthetic (metallic, alloy, ceramic, plastic, and others). Human body is a very aggressive corrosive environment and arising probability of the SCC or FC cracks is very high. However, evaluation of the $\mathrm{SC}$ cracks is quite difficult because they are partially conductive, Fig. 2.

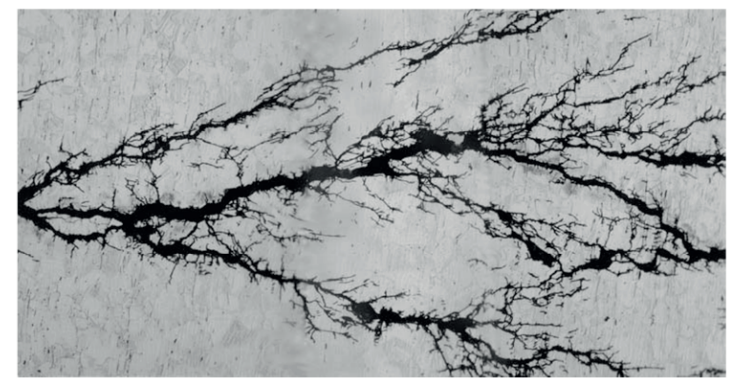

Fig. 2.Internal structure of the SC crack (after destructive evaluation)

\footnotetext{
* Milan Smetana, Klara Capova

Department of Electromagnetic and Biomedical Engineering, Faculty of Electrical Engineering, University of Zilina, Slovakia

E-mail: milan.smetana@fel.uniza.sk
} 


\section{Eddy current evaluation}

In recent years, electromagnetic methods, especially eddy current testing (ECT), have attracted increasing attention. The ECT method is theoretically well known and widely utilized in the practice. Eddy current testing is used to ensure preservice quality and to assess in-service health of industrial components made of electrically conducting materials, by way of detection and characterization of defects or discontinuities. Eddy current probe is the main link between the eddy current instrument and the component under test. Success of eddy current testing for a specific inspection application depends on sensor, instrument and optimization of test parameters. The probe plays two important roles: it induces the eddy currents, and it senses the distortion of their flow caused by defects. Design and development of eddy current probes is very important as it is the probe that dictates the probability of detection and the reliability of characterization, [3], [4], [5], [6] and [7]. Traditional eddy current testing method based on excitationdetection coils is fundamentally limited by the poor sensitivity of the detection coils at low frequencies, [8]. Nowadays comes to the fore the use of different types of detection elements such as Hall sensors, SQUID, GMR, AMR, Fluxgate and other to increase a detection sensitivity. The shape, cross-section, size and configuration of coils and sensing elements are varied to design an eddy current probe for a specific application, [4] and [5]. Among these, the magneto-resistive (MR) sensors offer a good trade-off in terms of performance versus cost. They have small dimensions, high sensitivity over a broad range of frequency (from hertz to megahertz domains), low noise; they operate at room temperature, and are inexpensive. It has been demonstrated that the MR probes perform better than conventional probes for low-frequency applications, e.g. when detecting deeply buried flaws. This is because the electromagnetic sensors are sensitive to the magnitude of the magnetic field. In the case of inductive-based probes, the output voltage is proportional to the time variation of the magnetic field; therefore, their sensitivity is reduced at low frequencies. Although their sensitivities are comparable, GMR sensors have better directional property than AMR sensors. Both types of sensors detect the component of the magnetic field vector along their sensitive axis. In the case of GMR sensors, fields applied perpendicularly to the sensitive axis have negligible effect on their output. In contrast, the sensitivity of AMR-based probes is lowered by a field perpendicular to the sensitive axis, which, at high values, can even "flip" the sensor response [4]. This property is particularly important in the coil-crack interaction problems where the electromagnetic field has a complex three-dimensional (3-D) distribution. The directional property of GMR sensor can be used in a difficult problem encountered in NDE, e. g, detection of edge cracks [8]. It is shown that by properly orienting the sensitive axis, the probe will be insensitive to the edge. Additionally, the presence of the edge enhances the sensitivity and resolution of the
GMR probe to cracks initiating perpendicular to this edge. The main objective in these strategies is to detect the weak magnetic fields from the defects rather than the traditional measurement of the impedance changes. When more than one sensor is used and data fusion methods are adopted to combine the sensors data then it forms a comprehensive global picture of investigated regions. This article deals with comparison of the four sensing elements: inductance coil, GMR, AMR and fluxgate sensor, in eddy current evaluation under the same conditions. The harmonic excitation is used to drive the eddy currents. The sensitivity and the resolution of the sensors are concerned. The reason for this comparison is to show which of these sensors is able to provide higher probability of detection and to give response signal with higher information rate for accurate characterization of defects.

\section{Experimental set-up}

The SUS 316L plate specimen with a thickness of $h_{1}=10 \mathrm{~mm}$ is inspected from the near-side. The material has the conductivity of $\sigma=1.4 \mathrm{MS} / \mathrm{m}$ and the relative permeability of $\mu_{\mathrm{r}}=1$. Every specimen contains one SCC and FC crack, located in the middle of the specimen, respectively, Fig. 3. The SCC cracks were made using corrosive environment $\left(\mathrm{MgCl}_{2}\right)$ and static load with various exposure times.

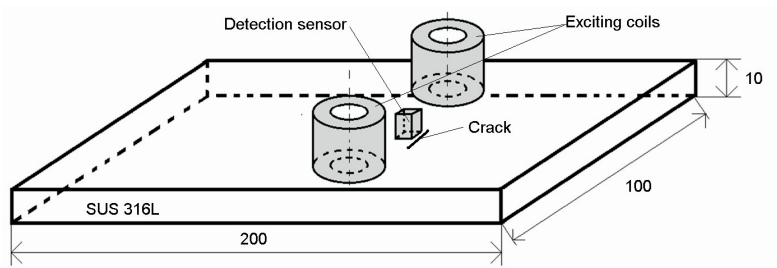

Fig. 3. Configuration of the plate specimen with the crack

New ECT probe is designed for this study. It consists of two exciting coils that are positioned normally to the surface of inspected material apart from each other. The coils are connected in series but magnetically opposite to decrease coupling between the exciting system and the sensing element. High sensitivity of a pick-up circuit can be adjusted in such case. The exciting coils are driven by the harmonic current with various frequencies. Four sensing elements with appropriate dimensions, the inductance coil, the fluxgate magnetometer, the GMR sensor and the AMR sensor, shown in Fig. 4, are used to pick-up the response signal.

The inductance coil is a classical type without the ferrite core. The fluxgate magnetometer is the commercial one made by Canon. The GMR and AMR sensor are the commercial ones made by Sensitec $\mathrm{GmbH}$. Measured component of the magnetic field in given direction is converted to the output voltage signal. Each inspection is performed four-times, once with the inductance coil and then with the fluxgate magnetometer and the GMR and AMR sensor, respectively. Realized modular probe with 
the sensing elements is shown in Fig. 5. Configuration of the experimental set-up is shown in Fig. 6 (SG - signal generator, PA power amplifier, EC - excitation coils, SD - sensing device, XYZ - linear positioning system, SC - stage controller, DAC - data acquisition card, LI - lock-in amplifier, PC - personal computer). a

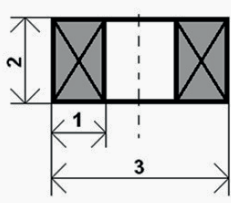

C

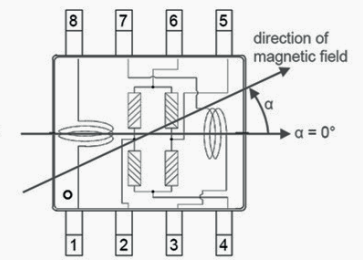

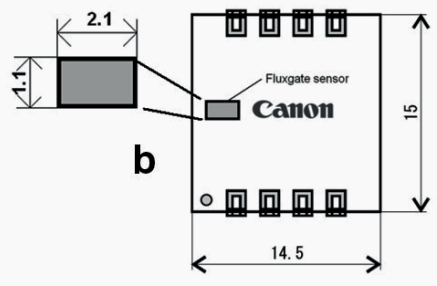

d

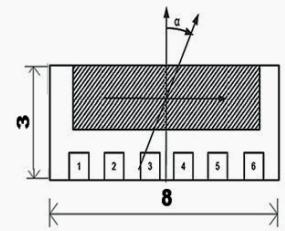

Fig. 4. Package outline of the sensing elements: inductance coil (a), fluxgate sensor (b), AMR sensor (c), GMR sensor (d)

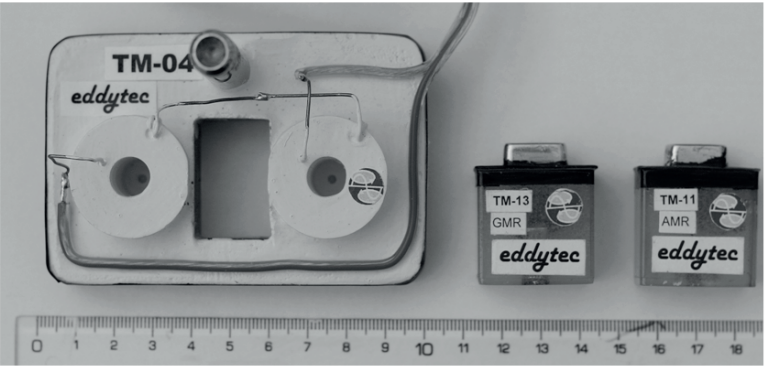

Fig. 5. Designed modular ECT probe (left) with the sensing elements (right)

Measured data are acquired using the data acquisition card with resolution of $16 \mathrm{bits} / \mathrm{channel}, 25 \mathrm{kS} / \mathrm{sec}$. User interface for data manipulation, controlling the stage and processing the data is created using the LabVIEW development environment. One dimensional scanning is performed over each crack along its length in a range $<-30 \mathrm{~mm}, 30 \mathrm{~mm}>$ relative to a crack center. The sensing elements are positioned in the middle between the exciting coils. Layout of the probe is shown in Fig. 7. Two exciting coils with self-inductances of $L_{1}=2.24 \mathrm{mH}, L_{2}=2.23 \mathrm{mH}$ are driven by the harmonic current with an effective value of $I=0.7$ A. The inductance receiver coil has $N=600$ number of turns and it is wound from a copper wire with a diameter of $\emptyset=0.05 \mathrm{~mm}$.

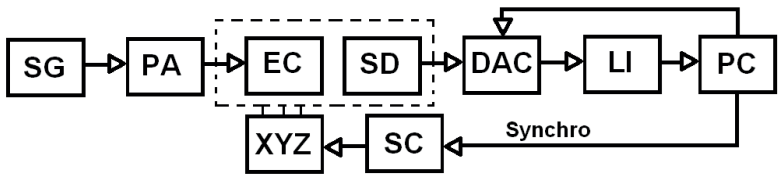

Fig. 6. Flowchart of the experimental setup

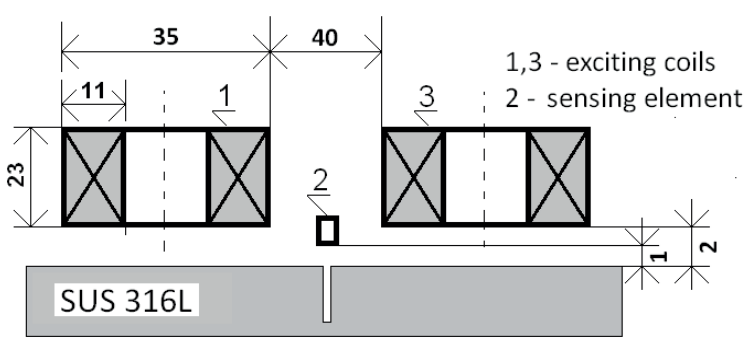

Fig. 7. Configuration of the designed and realized ECT probe

\section{Experimental results}

Results of the realized experiments are presented in this section. The SUS316L plate specimen with a thickness of $h=10 \mathrm{~mm}$ is inspected from the near-side. Figures $8-17$ display the sensors' response signals magnitude on the probe position relative to the crack center for the near side inspection. Several waveforms for different frequencies are displayed for every graph. It can be observed that the experimental results using various sensing elements have approximately comparable information value. The sensitivities of the sensors are quite similar when comparing the results in the relative scale. The individual signals for all the excitation frequencies are separated from each other using both types of sensing elements.

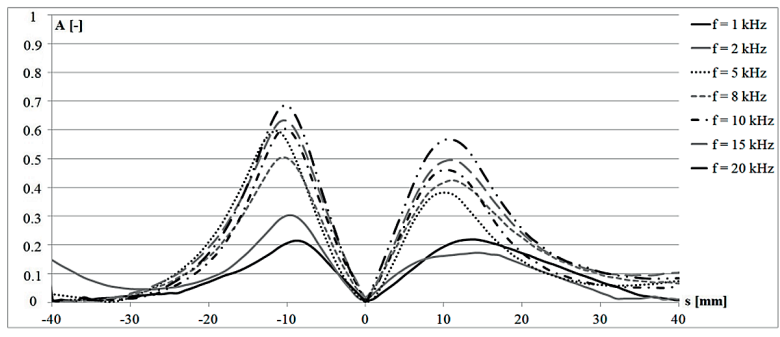

Fig. 8. Experimental results: GMR sensor, SC crack (load force $F=1.2 \mathrm{kN}, t=12$ hours)

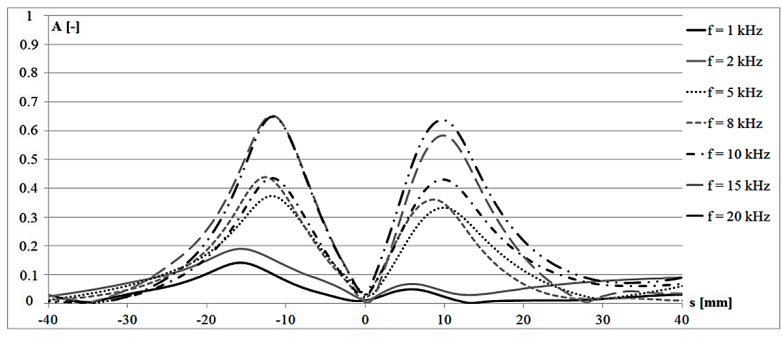

Fig. 9. Experimental results: inductance sensor, SC crack (load force $F=1.2 \mathrm{kN}, t=12$ hours)

Further, it can be observed that approximately the same resolution is obtained with all the magnetic field sensors. It 
seems that when an ECT probe is constructed in such a way that the direct coupling between the exciting system and the pick-up system is minimized all the sensing elements have comparable sensitivity and resolution. The results show that the higher the excitation frequency the higher the amplitude of the differential response signal.

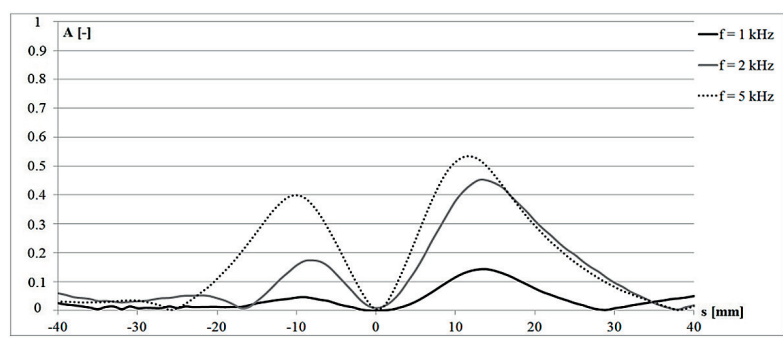

Fig. 10. Experimental results: fluxgate sensor, SC crack (load force $F=1.2 \mathrm{kN}, t=12$ hours)

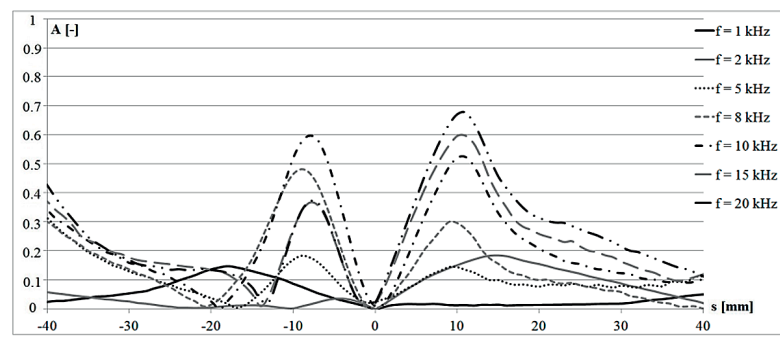

Fig. 11. Experimental results: GMR sensor, SC crack (load force $F=1.8 \mathrm{kN}, t=6$ hours)

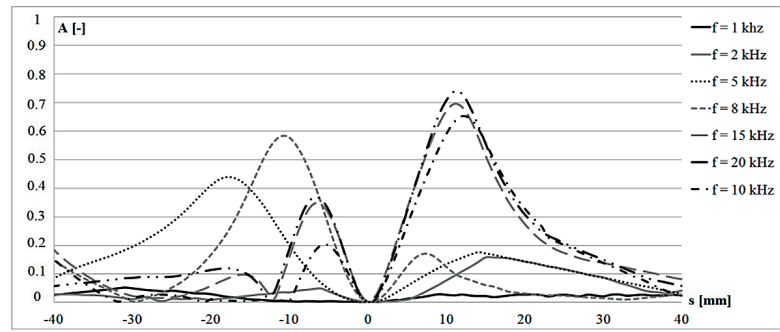

Fig. 12. Experimental results: inductance sensor, SC crack (load force $F=1.8 \mathrm{kN}, t=6$ hours)

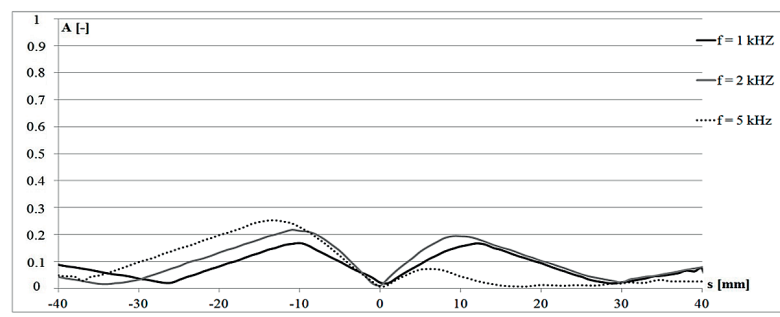

Fig. 13. Experimental results: fluxgate sensor, SC crack (load force $F=1.8 \mathrm{kN}, t=6$ hours)

The results for the fluxgate sensor represent only three individual waveforms because its upper frequency range was approximately $f_{\max }=3.4 \mathrm{kHz}$. All the sensed signals for one excitation frequency are shown in Fig. 16 to see the sensitivity of the sensors. It can be seen that the highest sensitivity was obtained using the GMR sensor. However, the length of the defect can be clearly detected observing the local peaks of the individual signals.

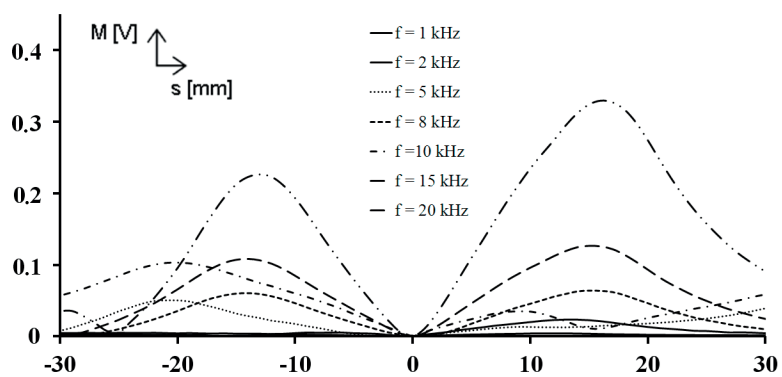

Fig. 14. Experimental results - AMR sensor, FC crack

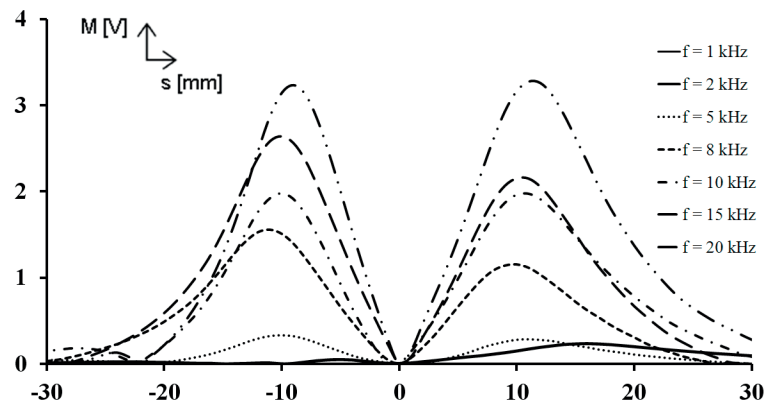

Fig. 15. Experimental results - GMR sensor, FC crack

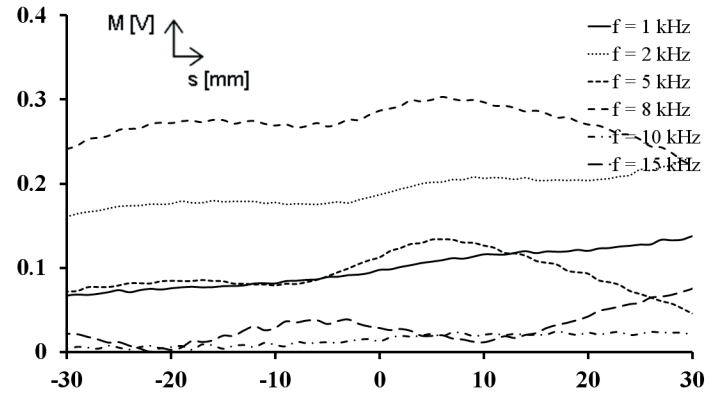

Fig. 16. Experimental results - AMR sensor (left), SCC crack (load force $F=1.8 \mathrm{kN}, t=12$ hours)

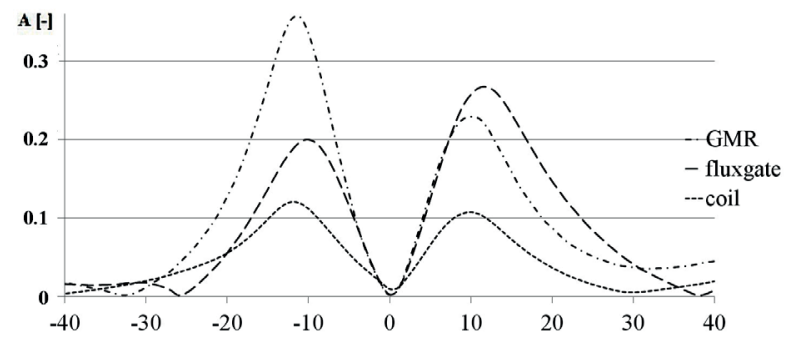

Fig. 17. Experimental results: SC crack,

(load force $F=1.2 \mathrm{kN}, t=24$ hours), excitation frequency $f=1 \mathrm{kHz}$ 


\section{Conclusion}

The article presented the design and performances of the eddy current probe in non-destructive inspection under harmonic excitation. Realized experiments were carried out to evaluate sensitivity and resolution of the four different sensors under the same conditions. Austenitic steel plates with presence of stress-corrosion crack and fatique crack were evaluated, respectively. According to the results it can be concluded that the four sensors have almost equal performances under the same conditions. Evaluated stress-corrosion and fatique cracks made under different conditions present real biomaterial's cracks that may occur in conductive prosthetic replacements in vivo. Because of complicated SCC's internal structure and partially conductive character, their detection and especially evaluation is quite difficult. On the other hand, based on the results, it can be seen that such types of material defects can be detected also at relatively low frequencies. Information value of the useful signals increase with higher excitation frequency. It can be concluded that even when the four different sensors exhibit almost similar performances, it is recommended to use the GMR sensor, because of its parameters (low noise, very high sensitivity in the senstive axis, cost etc. )

Further work of the authors will address the behaviour of other types of multi-axis sensing elements under various types of excitations.

\section{Acknowledgment}

The authors wish to express their thanks to prof. Peter Palcek and his co-workers of Department of Mechanical Engineering, University of Zilina, for the cooperation. This work was supported by the Slovak Research and Development Agency under the contracts No. APVV-0349-10.

\section{References}

[1] REIG, C., CUBBELS-BELTRAN, M. D., MUNOZ RAMIREZ, D.: Magnetic Field Sensors Based on Giant Magnetoresistance (GMR) Technology: Applications in Electrical Current Sensing, Sensors 2009, ISSN 1424-8220, pp. 7920 - 7924

[2] BETTA, G., FERRIGNO, L., LATACCA M.: GMR - Based ECT Instrument for Detection and Characterization of Crack on Planar Specimen: A Hand-held Solution, IEEE Transactions on Instrumentation and Measurement, vol. 61, No. 2, February 2012, pp. 505-512

[3] RIPKA, P.: Advances in Fluxgate Sensors, ScienceDirect, Sensors and Actuators A 106, 2003, pp. 8-14

[4] PERnisova, V., CAPOVA, K., SMETANA, M., STRAPACOVA, T.: Biomaterial Real Defects Evaluation Using Advanced Detection Sensors, Proc. of Intern. workshop on Electromagnetic non-destructive evaluation, 2013, Bratislava, ISBN 978-80-554-0713-5

[5] JANOUSEK, L.: Impact of Selected Parameters on Eddy Current Attenuation in Conductive Materials, 2012, Proc. of $9^{\text {th }}$ Intern. Conference, ELEKTRO 2012, art. No. 6225656

[6] JANOUSEK, L., REBICAN, M. I., SMETANA, M., STRAPACOVA, T., DUCA, A., PERNISOVA, V.: Recent Innovative Solutions in Eddy Current Non-destructive Diagnosis, Communications - Scientific Letters of the University of Zilina, vol. 15, No. 2, 2013, pp. 102-108, ISSN: 13354205

[7] STRAPACOVA, T., CAPOVA, K., SMETANA, M.: Biomaterials Inhomogeneities Detection by Electromagnetic Methods. Communications - Scientific Letters of the University of Zilina, vol. 13, No. 1, 2011, pp. 32-36, ISSN 1335-4205.

[8] JANOUSEK, L.: Effect of Exciting System Configuration on Eddy Currents Distribution in Non-destructive Evaluation of Materials, 2013, Przeglad Elektrotechniczny 89(3A), pp. 256-258. 